PROCEEDINGS OF THE

AMERICAN MATHEMATICAL SOCIETY

Volume 127, Number 11, Pages 3131-3140

S 0002-9939(99)05154-0

Article electronically published on May 4, 1999

\title{
NORMALIZERS OF THE CONGRUENCE SUBGROUPS OF THE HECKE GROUP $G_{5}$
}

\author{
MONG-LUNG LANG AND SER-PEOW TAN
}

(Communicated by Ronald M. Solomon)

\begin{abstract}
Let $\lambda=2 \cos (\pi / 5)$ and let $G$ be the Hecke group associated to $\lambda$. In this article, we show that for $\tau$ a prime ideal in $\mathbb{Z}[\lambda]$, the congruence subgroups $G_{0}(\tau)$ of $G$ are self-normalized in $P S L_{2}(\mathbb{R})$.
\end{abstract}

\section{INTRODUCTION}

In this paper, we continue our study into the extent to which properties of the modular group hold for the Hecke groups; see [CLLT], [LLT1], [LLT2] for some previous results. We are, in particular, interested in the Hecke group $G_{5}$ which we denote by $G$ and its congruence subgroups $G_{0}(\tau)$ of prime level $\tau$. Our main result is that the groups $G_{0}(\tau)$ are self-normalized in $P S L_{2}(\mathbb{R})$. This contrasts with the case of the congruence subgroups $\Gamma_{0}(p)$ of the modular group $\Gamma$ which admit Atkin-Lehner involutions, so have strictly larger normalizers; see for example [AL].

We recall the following definitions, notation and results. For $q \geq 4$, the Hecke groups $G_{q}$ are the (discrete) subgroups $\left\langle w, u_{q}\right\rangle$ of $P S L_{2}\left(\mathbb{Z}\left[\lambda_{q}\right]\right)$ where $\lambda_{q}=2 \cos (\pi / q)$ and

$$
w=\left(\begin{array}{cc}
0 & -1 \\
1 & 0
\end{array}\right), u_{q}=\left(\begin{array}{cc}
1 & \lambda_{q} \\
0 & 1
\end{array}\right) .
$$

When $q=3$, we recover the modular group $\Gamma$ so the above can be thought of as a natural generalization of $\Gamma$. Alternatively, we can interpret the generalization as $G_{q}$ being maximal discrete subgroups whose entries are in some extension of $\mathbb{Z}$. Finally, we have the geometric interpretation: $\Gamma$ is a $(2,3, \infty)$ triangle group and the Hecke group $G_{q}$ is a $(2, q, \infty)$ triangle group.

Let $\mathcal{A}$ be an ideal of $\mathbb{Z}\left[\lambda_{q}\right]$. We define

$$
G_{0}(\mathcal{A})=\left\{\sigma=\left(\begin{array}{ll}
a & b \\
c & d
\end{array}\right) \in G_{q} \mid c \in \mathcal{A}\right\} .
$$

Again, this is a natural generalization of the congruence subgroups $\Gamma_{0}(n)$ of $\Gamma$. It works because the elements of $G_{q}$ sit naturally in the ring $\mathbb{Z}\left[\lambda_{q}\right]$.

Recall that $G_{q}$ is commensurable with $P S L_{2}(\mathbb{Z})$ if and only if $q=4$ or 6 . The elements of such groups are completely known; see $[\mathrm{P}]$, for example. The normalizer of $G_{0}(\mathcal{A})$ in $P S L_{2}(\mathbb{R})$ can be determined [LT].

Received by the editors January 10, 1998.

1991 Mathematics Subject Classification. Primary 11F06.

Key words and phrases. Congruence subgroups, Hecke groups.

(C)1999 American Mathematical Society 
Suppose $G_{q}$ is not commensurable with $P S L_{2}(\mathbb{Z})$. By the results of Leutbecher, [L1], [L2], $\mathbb{Q}[\lambda] \cup\{\infty\}$ is the set of cusps of $G_{q}$ if and only if $q=5$. Also, 5 is the only $q$ other than 4,6 for which $\mathbb{Q}[\lambda]$ is a quadratic field. For all other $q$ 's, the degree is $>2$. As a consequence, $q=5$ is the next most workable and interesting $q$. Some of the classical results on the modular group can be generalized to $G=G_{5}$ ([CLLT], [LLT2]). The main result in this paper is the following:

Main Theorem. If $(\tau)$ is a prime ideal of $\mathbb{Z}[\lambda]=\mathbb{Z}\left[\lambda_{5}\right]$, then $G_{0}(\tau) \leq G_{5}=G$ is self-normalized in $\mathrm{PSL}_{2}(\mathbb{R})$.

The main facts used in the proof are:

(a) $\mathbb{Z}[\lambda]$ is a principal ideal domain.

(b) The set of cusps of $G$ is $\mathbb{Q}[\lambda] \cup\{\infty\}$ ([L1], [L2]). Furthermore, if $x \in \mathbb{Q}[\lambda]$ is a cusp, $x$ has a unique reduced form $x=\frac{a}{b}$ [LLT1]. By definition, this means that $a, b \in \mathbb{Z}[\lambda]$ with $b>0$ and there exists $c, d \in \mathbb{Z}[\lambda]$ such that $\left(\begin{array}{ll}a & c \\ b & d\end{array}\right) \in G$. Clearly, $(a, b)=1$ so that if $x=\frac{a^{\prime}}{b^{\prime}}$ with $\left(a^{\prime}, b^{\prime}\right)=1$, then $a=\mu a^{\prime}, b=\mu b^{\prime}$ where $\mu$ is a unit in $\mathbb{Z}[\lambda]$.

(c) (Proposition 6 of [LLT1]) Suppose $x_{i}, x_{j}$ are $G$-rationals with reduced form $a_{i} / b_{i}$ and $a_{j} / b_{j}$, respectively, and suppose that $x_{i}<x_{j}$. Then the following statements are equivalent:

(i) $\left(\begin{array}{ll}a_{i} & a_{j} \\ b_{i} & b_{j}\end{array}\right) \in G$;

(ii) $\left(x_{i}, x_{j}\right)$ is an even line, that is, it is the image of the complete hyperbolic geodesic with ends at 0 and $\infty$ under the action of some $A \in G$;

(iii) $a_{j} b_{i}-a_{i} b_{j}=1$.

(d) (Corollary 5 of [LLT1]) $\left(\begin{array}{ll}1 & b \\ 0 & 1\end{array}\right) \in G$ if and only if $b=m \lambda, m \in \mathbb{Z}$. Similarly, $\left(\begin{array}{ll}1 & 0 \\ c & 1\end{array}\right) \in G$ if and only if $c=n \lambda, n \in \mathbb{Z}$.

The rest of this paper is organized as follows. In the next section, we give the possible forms which an element $A$ of $N\left(G_{0}(\tau)\right)$ can take, breaking it into different cases. In section 3 , we complete the proof of the main theorem in the case where the group $G_{0}(\tau)$ has 2 inequivalent cusps. By [CLLT], this occurs when the rational prime $p$ lying under $\tau$ is 5 or is congruent to $\pm 1(\bmod 10)$. Finally, in section 4 , we complete the proof of the main theorem in the case where the group $G_{0}(\tau)$ has $p+1$ cusps. By [CLLT], this occurs when the rational prime $p$ lying under $\tau$ is congruent to $\pm 3(\bmod 10)$ or $p=2$.

\section{UPPER BOUND FOR $N\left(G_{0}(\tau)\right)$}

Let $I$ be a prime in $\mathbb{Z}[\lambda]=\mathbb{Z}\left[\lambda_{5}\right]$. Since $\mathbb{Z}[\lambda]$ is a principal ideal domain, $I=(\tau)$ for some $\tau$. Note that we may assume that $\tau$ is positive. Let $p$ be the positive rational prime which lies below $\tau$. It is an easy matter to check that $p$ is square free in $\mathbb{Z}[\lambda]$ if and only of $p \neq 5$.

Denote by $N\left(G_{0}(\tau)\right)$ the normalizer of $G_{0}(\tau)$ in $P S L_{2}(\mathbb{R})$. For any

$$
A=\left(\begin{array}{ll}
a & b \\
c & d
\end{array}\right) \in N\left(G_{0}(\tau)\right),
$$


we have

$$
\begin{aligned}
\left(\begin{array}{ll}
a & b \\
c & d
\end{array}\right)\left(\begin{array}{ll}
1 & \lambda \\
0 & 1
\end{array}\right)\left(\begin{array}{ll}
a & b \\
c & d
\end{array}\right)^{-1}=\left(\begin{array}{cc}
1-a c \lambda & a^{2} \lambda \\
-c^{2} \lambda & 1+a c \lambda
\end{array}\right) \in G_{0}(\tau), \\
\left(\begin{array}{ll}
a & b \\
c & d
\end{array}\right)^{-1}\left(\begin{array}{ll}
1 & \lambda \\
0 & 1
\end{array}\right)\left(\begin{array}{ll}
a & b \\
c & d
\end{array}\right)=\left(\begin{array}{cc}
1+d c \lambda & d^{2} \lambda \\
-c^{2} \lambda & 1-d c \lambda
\end{array}\right) \in G_{0}(\tau), \\
\left(\begin{array}{ll}
a & b \\
c & d
\end{array}\right)\left(\begin{array}{cc}
1 & 0 \\
p \lambda & 1
\end{array}\right)\left(\begin{array}{ll}
a & b \\
c & d
\end{array}\right)^{-1}=\left(\begin{array}{cc}
1+b d p \lambda & -b^{2} p \lambda \\
d^{2} p \lambda & 1-b d p \lambda
\end{array}\right) \in G_{0}(\tau), \\
\left(\begin{array}{ll}
a & b \\
c & d
\end{array}\right)^{-1}\left(\begin{array}{cc}
1 & 0 \\
p \lambda & 1
\end{array}\right)\left(\begin{array}{ll}
a & b \\
c & d
\end{array}\right)=\left(\begin{array}{cc}
1-a b p \lambda & -b^{2} p \lambda \\
a^{2} p \lambda & 1+a b p \lambda
\end{array}\right) \in G_{0}(\tau) .
\end{aligned}
$$

In the rest of this section, we determine the possible forms that $A$ can take (Lemmas 1-5).

Lemma 1. $N\left(G_{0}(\tau)\right) \cap P S L_{2}(\mathbb{Z}[\lambda])=G_{0}(\tau)$.

Proof. For any $A \in N\left(G_{0}(\tau)\right) \cap P S L_{2}(\mathbb{Z}[\lambda])$, by $(2.1)$, the $(2,1)$-entry of $A$ is a multiple of $\tau$. Hence

$$
A=\left(\begin{array}{cc}
a & b \\
c \tau & d
\end{array}\right)
$$

where $a, b, c, d \in \mathbb{Z}[\lambda]$. Suppose $c \neq 0$. Recall that $x=a / c \tau \in \mathbb{Q}(\lambda)$ is a cusp of $G$ [L1], [L2]. Let $x=a^{\prime} / c^{\prime}$ be the reduced form for $x$. $G$ contains an element of the form

$$
B=\left(\begin{array}{ll}
a^{\prime} & b^{\prime} \\
c^{\prime} & d^{\prime}
\end{array}\right)
$$

Since $(a, c \tau)=1, c^{\prime}=\mu c \tau$ where $\mu$ is a unit of $\mathbb{Z}[\lambda]$. Hence $c^{\prime}$ is a multiple of $\tau$. This implies that $B \in G_{0}(\tau) \leq N\left(G_{0}(\tau)\right)$. Since $A \infty=B \infty$, it follows that

$$
B^{-1} A=\left(\begin{array}{cc}
u & v \\
0 & u^{-1}
\end{array}\right) \in N\left(G_{0}(\tau)\right)
$$

where $u, v \in \mathbb{Z}[\lambda]$. Applying (2.1) and (2.2) to $B^{-1} A$, one has that

$$
\left(\begin{array}{cc}
1 & u^{2} \lambda \\
0 & 1
\end{array}\right),\left(\begin{array}{cc}
1 & u^{-2} \lambda \\
0 & 1
\end{array}\right)
$$

are elements of $G_{0}(\tau)$. By Corollary 5 of [LLT1], $u= \pm 1$. Multiplying $B^{-1} A$ by $-I$ if necessary, we may assume that $u=1$ and

$$
B^{-1} A=\left(\begin{array}{cc}
1 & x+y \lambda \\
0 & 1
\end{array}\right)
$$

where $x, y \in \mathbb{Z}$. Note that

$$
\left(\begin{array}{cc}
1 & y \lambda \\
0 & 1
\end{array}\right) \in N\left(G_{0}(\tau)\right)
$$

As a consequence,

$$
\left(\begin{array}{ll}
1 & x \\
0 & 1
\end{array}\right) \in N\left(G_{0}(\tau)\right)
$$


Suppose that $x \neq 0$. Since $\lambda \in \mathbb{R} \backslash \mathbb{Q}$, for any $\epsilon>0$, there exist $k$ and $l$ such that

$$
\left(\begin{array}{ll}
1 & x \\
0 & 1
\end{array}\right)^{k}\left(\begin{array}{ll}
1 & \lambda \\
0 & 1
\end{array}\right)^{l}=\left(\begin{array}{ll}
1 & \delta \\
0 & 1
\end{array}\right)=\sigma \in N\left(G_{0}(\tau)\right)
$$

where $0<|\delta|<\epsilon$. As a consequence,

$$
\sigma\left(\begin{array}{cc}
1 & 0 \\
p \lambda & 1
\end{array}\right) \sigma^{-1}=\left(\begin{array}{cc}
1+\delta p \lambda & \delta^{2} p \lambda \\
p \lambda & 1-\delta p \lambda
\end{array}\right) \in G_{0}(\tau) .
$$

This implies that $G_{0}(\tau)$ is not discrete, giving a contradiction. Hence $x=0$ and $B^{-1} A \in G_{0}(\tau)$. Since $B \in G_{0}(\tau), A \in G_{0}(\tau)$.

Suppose $c=0$. From the above argument, we have $A \in G_{0}(\tau)$.

Lemma 2. Suppose $p \neq 5, A \in N\left(G_{0}(\tau)\right)$. If $c=0$, then $A \in G_{0}(\tau)$.

Proof. Applying (2.1), (2.2), (2.3), and (2.4) to $A$, we have

$$
\left(\begin{array}{cc}
1 & a^{2} \lambda \\
0 & 1
\end{array}\right),\left(\begin{array}{cc}
1 & d^{2} \lambda \\
0 & 1
\end{array}\right),\left(\begin{array}{cc}
1+b d p \lambda & -b^{2} p \lambda \\
d^{2} p \lambda & 1-b d p \lambda
\end{array}\right),\left(\begin{array}{cc}
1-a b p \lambda & -b^{2} p \lambda \\
a^{2} p \lambda & 1+a b p \lambda
\end{array}\right)
$$

are elements of $G_{0}(\tau)$. By Corollary 5 of [LLT1], $a^{2}$ and $d^{2}$ are elements in $\mathbb{Z}$. Since $a d=1, a=d= \pm 1$. Multiplying by $-I$ if necessary, we may assume that $a=d=1$. Since $b d p=b p \in \mathbb{Z}[\lambda], b=k / p$ for some $k \in \mathbb{Z}[\lambda]$. Since $b^{2} p \in \mathbb{Z}[\lambda]$, $k^{2} / p \in \mathbb{Z}[\lambda]$. It follows that $k$ is a multiple of $p$ ( $p$ is square free). Consequently, $b=k / p=x+y \lambda \in \mathbb{Z}[\lambda]$ and $A$ is of the form

$$
A=\left(\begin{array}{cc}
1 & x+y \lambda \\
0 & 1
\end{array}\right)
$$

Applying the proof of Lemma 1, we conclude that $A \in G_{0}(\tau)$.

Remark. If $p=5, \tau=2+\lambda$ and by direct calculation, we can show that if $c=0$, then $A$ is of the form $A=\left(\begin{array}{cc}1 & k / \sqrt{5} \\ 0 & 1\end{array}\right)$, where $k \in \mathbb{Z}[\lambda]$.

Lemma 3. Suppose $A \in N\left(G_{0}(\tau)\right)$. If $b=0$, then $A \in G_{0}(\tau)$.

Proof. Applying (2.1), (2.2), (2.3) and (2.4) to $A$, we have

$$
\left(\begin{array}{cc}
1-a c \lambda & a^{2} \lambda \\
-c^{2} \lambda & 1+a c \lambda
\end{array}\right),\left(\begin{array}{cc}
1+d c \lambda & d^{2} \lambda \\
-c^{2} \lambda & 1-d c \lambda
\end{array}\right),\left(\begin{array}{cc}
1 & 0 \\
d^{2} p \lambda & 1
\end{array}\right),\left(\begin{array}{cc}
1 & 0 \\
a^{2} p \lambda & 1
\end{array}\right)
$$

are elements of $G_{0}(\tau)$. By Corollary 5 of [LLT1], $a^{2}$ and $d^{2}$ are elements in $\mathbb{Z}$. Since $a d=1, a=d= \pm 1$. As a consequence, $c \in \mathbb{Z}[\lambda]$. This implies that $A \in P S L_{2}(\mathbb{Z}[\lambda])$. By Lemma $1, A \in G_{0}(\tau)$.

Lemma 4. Suppose $p \neq 5$. Let $A \in N\left(G_{0}(\tau)\right)$. Then $A$ is of the form

$$
\left(\begin{array}{cc}
0 & -1 / \sqrt{p} \\
\sqrt{p} & h \sqrt{p}
\end{array}\right) \text { if } a=0,\left(\begin{array}{cc}
h \sqrt{p} & 1 / \sqrt{p} \\
-\sqrt{p} & 0
\end{array}\right) \text { if } d=0
$$

where $h \in \mathbb{Z}[\lambda]$.

Proof. Suppose $a=0$. Applying (2.1), (2.2), (2.3) and (2.4) to $A$, we have,

$$
\left(\begin{array}{cc}
1 & 0 \\
-c^{2} \lambda & 1
\end{array}\right),\left(\begin{array}{cc}
1+d c \lambda & d^{2} \lambda \\
-c^{2} \lambda & 1-d c \lambda
\end{array}\right),\left(\begin{array}{cc}
1+b d p \lambda & -b^{2} p \lambda \\
d^{2} p \lambda & 1-b d p \lambda
\end{array}\right),\left(\begin{array}{cc}
1 & -b^{2} p \lambda \\
0 & 1
\end{array}\right)
$$

are elements of $G_{0}(\tau)$. By Corollary 5 of [LLT1], $c^{2}=k p$ for some $k \in \mathbb{Z}, b^{2} p=l \in$ $\mathbb{Z}$. Hence $c^{2} b^{2} p=l k p, k \in \mathbb{Z}$. Since $b c=-1$, one has $k=1, l=1$. It follows that 
$c= \pm \sqrt{p}, b=\mp 1 / \sqrt{p}$. Multiplying by $-I$ if necessary, we may assume that $c=\sqrt{p}$. Since $d c \in \mathbb{Z}[\lambda], d=s / \sqrt{p}$ for some $s \in \mathbb{Z}[\lambda]$. Since $d^{2} \in \mathbb{Z}[\lambda], d^{2}=s^{2} / p \in \mathbb{Z}[\lambda]$. Since $p$ is square free $(p \neq 5), s=h p$ for some $h \in \mathbb{Z}[\lambda]$. Hence $d=h \sqrt{p}$ and

$$
A=\left(\begin{array}{cc}
0 & -1 / \sqrt{p} \\
\sqrt{p} & h \sqrt{p}
\end{array}\right) .
$$

Suppose $d=0$. Applying (2.1), (2.2), (2.3) and (2.4) to $A^{-1}$, we have,

$$
A^{-1}=\left(\begin{array}{cc}
0 & -1 / \sqrt{p} \\
\sqrt{p} & h \sqrt{p}
\end{array}\right) .
$$

This completes the proof of the lemma.

Remark. If $p=5$, by direct calculation, we can show that $\mathrm{A}$ is of the form

$$
\left(\begin{array}{cc}
0 & -1 / \sqrt{5} \\
\sqrt{5} & h
\end{array}\right) \text { if } a=0,\left(\begin{array}{cc}
h & 1 / \sqrt{5} \\
-\sqrt{5} & 0
\end{array}\right) \text { if } d=0 .
$$

Lemma 5. Let $A \in N\left(G_{0}(\tau)\right)$. Suppose $p \neq 5$ and abcd $\neq 0$. Then either $A \in$ $G_{0}(\tau)$ or $b / a \in \mathbb{Q}(\lambda)$ and the denominator of the reduced form of $b / a$ is a multiple of $\tau$.

Proof. By (2.1), (2.2), (2.3) and (2.4),

$$
\begin{gathered}
\left(\begin{array}{cc}
1-a c \lambda & a^{2} \lambda \\
-c^{2} \lambda & 1+a c \lambda
\end{array}\right),\left(\begin{array}{cc}
1+d c \lambda & d^{2} \lambda \\
-c^{2} \lambda & 1-d c \lambda
\end{array}\right), \\
\left(\begin{array}{cc}
1+b d p \lambda & -b^{2} p \lambda \\
d^{2} p \lambda & 1-b d p \lambda
\end{array}\right),\left(\begin{array}{cc}
1-a b p \lambda & -b^{2} p \lambda \\
a^{2} p \lambda & 1+a b p \lambda
\end{array}\right)
\end{gathered}
$$

are elements of $G_{0}(\tau)$. By (2.1), $c^{2} \in \mathbb{Z}[\lambda]$ is a multiple of $\tau$. It follows easily that one of the following holds:

(a) $c=s \tau$ where $s \in \mathbb{Z}[\lambda]$,

(b) $c=s \sqrt{\tau}$ where $s \in \mathbb{Z}[\lambda]$,

(c) $c=s \tau \sqrt{w}$ where $s, w \in \mathbb{Z}[\lambda]((w, \tau)=1), w$ is square free,

(d) $c=s \sqrt{w \tau}$ where $(w, \tau)=1$ and $s, w \in \mathbb{Z}[\lambda], w$ is square free.

(a) By (2.1) $a c \in \mathbb{Z}[\lambda]$. This implies that $a=r / s \tau$, where $r \in \mathbb{Z}[\lambda]$. By (2.1) $a^{2}=(r / s \tau)^{2} \in \mathbb{Z}[\lambda]$. It follows that $r$ is a multiple of $s \tau$. Hence $a=r / s \tau \in \mathbb{Z}[\lambda]$. By $(2.2) d c, d^{2} \in \mathbb{Z}[\lambda]$, similar to the above, $d \in \mathbb{Z}[\lambda]$. By (2.3) $b d p \in \mathbb{Z}[\lambda]$. Hence $b=t / d p$ for some $t \in \mathbb{Z}[\lambda]$. Since $b^{2} p \in \mathbb{Z}[\lambda], t^{2} / d^{2} p \in \mathbb{Z}[\lambda]$. Since $p$ is square free, $p d$ is a divisor of $t$. This implies that $b \in \mathbb{Z}[\lambda]$. Summing up the above, we have $A \in N\left(G_{0}(p)\right) \cap P S L_{2}(\mathbb{Z}[\lambda])=G_{0}(p)$ (Lemma 1).

(b) By (2.1) $a c \in \mathbb{Z}[\lambda]$. This implies that $a=r^{\prime} / s \sqrt{\tau}$, where $r^{\prime} \in \mathbb{Z}[\lambda]$. By (2.1) $a^{2}=\left(r^{\prime} / s \sqrt{\tau}\right)^{2}=r^{\prime 2} / s^{2} \tau \in \mathbb{Z}[\lambda]$. Since $\tau$ is a prime, It follows that $s \tau \mid r^{\prime}$. Hence $a=r \sqrt{\tau}$ where $r \in \mathbb{Z}[\lambda]$. By $(2.2) d c, d^{2} \in \mathbb{Z}[\lambda]$. Using a similar argument to the above, we have $d=u \sqrt{\tau}$ where $u \in \mathbb{Z}[\lambda]$. By (2.3) bdp $\in \mathbb{Z}[\lambda]$. Hence $b=t^{\prime} / d p=t^{\prime} / u p \sqrt{\tau}$ for some $t^{\prime} \in \mathbb{Z}[\lambda]$. Since $b^{2} p \in \mathbb{Z}[\lambda], b^{2} p=t^{\prime 2} / u^{2} p \tau \in \mathbb{Z}[\lambda]$. Hence $p u \mid t^{\prime}$. This implies that $b=t / \sqrt{\tau}$ where $t \in \mathbb{Z}[\lambda]$. Summing up the above, we conclude that $b / a=t / \tau r \in \mathbb{Q}(\lambda)$. Since $r u \tau-s t=1,(t, \tau)=1$. It follows that the denominator of the reduced form of $b / a$ is a multiple of $\tau$.

(c) Let $p=\tau \tau^{\prime}$. As in (b), we may show

$$
a=r \sqrt{w}, b=t \sqrt{w}, d=u \sqrt{w}, \quad \text { if } \quad\left(\tau^{\prime}, w\right)=1
$$


and

$$
a=r \sqrt{w_{1} \tau^{\prime}}, b=t \sqrt{w_{1}} / \sqrt{\tau^{\prime}}, d=u \sqrt{w_{1} \tau^{\prime}}, \quad \text { if } \quad\left(\tau^{\prime}, w\right)=\tau^{\prime} \neq 1, w=w_{1} \tau^{\prime},
$$

where $r, t, u, w_{1} \in \mathbb{Z}[\lambda]$.

Suppose $\left(\tau^{\prime}, w\right)=1$. Since the determinant of $A$ is $1, w$ is a unit. We have $a / c=r / s \tau$. Let $X / Y$ be the reduced form of $a / c$. Since $r u w-s w t \tau=1,(r, s \tau)=1$. Hence $Y=\mu s \tau$ where $\mu$ is a unit of $\mathbb{Z}[\lambda]$. Since $X / Y$ is the reduced form of a cusp, $G$ admits an element of the form

$$
B=\left(\begin{array}{cc}
X & Z \\
Y & W
\end{array}\right) .
$$

Since $Y$ is a multiple of $\tau, B \in G_{0}(\tau)$. Since $A \infty=B \infty, B^{-1} A$ fixes $\infty$ and takes the following form:

$$
B^{-1} A=\left(\begin{array}{ll}
* & * \\
0 & *
\end{array}\right) \in N\left(G_{0}(\tau)\right) .
$$

By Lemma $2, B^{-1} A \in G_{0}(\tau)$. Hence $A \in G_{0}(\tau)$.

Suppose $w=w_{1} \tau^{\prime}$. Similar to the case $\left(w, \tau^{\prime}\right)=1, w_{1}$ is a unit in $\mathbb{Z}[\lambda]$. The denominator of the reduced form of $a / c=r / s \tau$ is again a multiple of $\tau$ and we have $A \in G_{0}(\tau)$. This gives a contradiction as $\sqrt{\tau^{\prime}} \notin \mathbb{Z}[\lambda]$.

(d) As in (c), we may show

$$
\begin{gathered}
a=r \sqrt{w \tau}, b=t \sqrt{w} / \sqrt{\tau}, d=u \sqrt{w \tau}, \quad \text { if } \quad(w, \tau)=1, \\
a=r \sqrt{w_{1} p}, b=t \sqrt{w_{1}} / \sqrt{p}, d=u \sqrt{w_{1} p}, \quad \text { if } \quad\left(\tau^{\prime}, w\right)=\tau^{\prime} \neq 1, w=w_{1} \tau^{\prime} .
\end{gathered}
$$

Using a similar argument to that in case (b), we can show that the denominator of the reduced form of $b / a$ is a multiple of $\tau$.

\section{Two Cusps}

In this section, we deal with the case when $G_{0}(\tau)$ has exactly 2 inequivalent cusps. By [CLLT], the prime $p$ lying below $\tau$ is 5 or $p \equiv \pm 1(\bmod 10)$ and $(p) \neq(\tau)$.

Theorem 6. Let $\tau$ be a prime such that $G_{0}(\tau)$ has exactly 2 inequivalent cusps. Then $N\left(G_{0}(\tau)\right)=G_{0}(\tau)$.

Proof. We first consider the case $p \neq 5$. Suppose $N\left(G_{0}(\tau)\right) \neq G_{0}(\tau)$. Let $A \in$ $N\left(G_{0}(\tau)\right) \backslash G_{0}(\tau)$. Suppose $A \infty$ is equivalent to $\infty$ in $G_{0}(\tau)$. Without loss of generality, we may assume $A \infty=\infty$. This implies that $c=0$ and $A \in G_{0}(\tau)$ (Lemma 2), a contradiction. Hence we may assume that $A \infty$ is not equivalent to $\infty$ in $G_{0}(\tau)$. Since $G_{0}(\tau)$ has exactly 2 inequivalent cusps, $A \infty$ is equivalent to 0 . Without loss of generality, we may assume that $A \infty=0$. It follows by Lemma 4 that $A$ is of the form

$$
A=\left(\begin{array}{cc}
0 & -1 / \sqrt{p} \\
\sqrt{p} & h \sqrt{p}
\end{array}\right) .
$$

Let $x=1 / \tau \in \mathbb{Q}(\lambda)$. By Leutbecher's theorem ([L1], [L2]), $x$ is a cusp of $G$. By [LLT1], the reduced form for $x$ is of the form $c / c \tau$, where $c$ is a unit in $\mathbb{Z}[\lambda]$. Consequently (Proposition 6(ii) of [LLT1]), $G_{0}(\tau)$ contains an element of the form

$$
B=\left(\begin{array}{cc}
c & b \\
c \tau & d
\end{array}\right)
$$


Since $A \in N\left(G_{0}(\tau)\right)$,

$$
A B A^{-1}=\left(\begin{array}{cc}
* & -c \tau / p \\
* * & *
\end{array}\right) \in G_{0}(\tau) .
$$

In particular, $-c \tau / p \in \mathbb{Z}[\lambda]$. This is a contradiction ( $c$ and $p$ have no common divisors in $\mathbb{Z}[\lambda]$ and $(\tau) \neq(p))$. It follows that $N\left(G_{0}(\tau)\right)=G_{0}(\tau)$.

Now, suppose $p=5$ and $N\left(G_{0}(\tau)\right) \neq G_{0}(\tau)$. In this case, $\tau=\lambda+2$. Let $A \in N\left(G_{0}(\tau)\right) \backslash G_{0}(\tau) . \quad A \infty$ is equivalent to either $\infty$ or 0 . Without loss of generality, we may assume that either $A \infty=\infty$ or $A \infty=0$. By the remarks following Lemma 2 and Lemma $4, A$ takes the form

$$
\begin{gathered}
A_{1}=\left(\begin{array}{cc}
1 & k / \sqrt{5} \\
0 & 1
\end{array}\right) \quad \text { if } \quad A \infty=\infty, \\
A_{2}=\left(\begin{array}{cc}
0 & -1 / \sqrt{5} \\
\sqrt{5} & h
\end{array}\right) \quad \text { if } \quad A \infty=0,
\end{gathered}
$$

where $h, k \in \mathbb{Z}[\lambda]$. By [LLT1],

$$
\sigma=\left(\begin{array}{cc}
\lambda & -1 \\
\lambda+2 & -\lambda
\end{array}\right) \in G_{0}(\lambda+2) .
$$

In the first case, since $A_{1} \sigma A_{1}^{-1} \in G_{0}(\lambda+2), k=\sqrt{5} u$ where $u \in \mathbb{Z}[\lambda]$. By Lemma $1, A_{1} \in G_{0}(\lambda+2)$, a contradiction. In the second case, an easy calculation shows that $A_{2} \sigma A_{2}^{-1} \notin G_{0}(\lambda+2)$, again giving a contradiction. It follows that $N\left(G_{0}(\lambda+2)\right)=G_{0}(\lambda+2)$.

$$
\text { 4. } p+1 \text { CUSPS }
$$

In this section, we assume that $p \equiv \pm 3(\bmod 10)$ or $p=2$. Then $p$ is prime in $\mathbb{Z}[\lambda]$ so $\tau=p$. By [CLLT], $G_{0}(\tau)$ has $p+1$ inequivalent cusps.

Lemma 7. Suppose $p \equiv \pm 3(\bmod 10)$. Then $-\lambda /(1+h p \lambda) \in \mathbb{Q}(\lambda)$ is in reduced form if and only if $h=k \lambda$ for some $k \in \mathbb{Z}$. If $p=2,-\lambda /(1+2 h \lambda) \in \mathbb{Q}(\lambda)$ is in reduced form if and only if $h=k \lambda$ or $1+k \lambda$ for some $k \in \mathbb{Z}$.

Proof. $-\lambda /(1+h m \lambda) \in \mathbb{Q}(\lambda)$ is in reduced form if and only if $(1+h m \lambda) / \lambda$ is in reduced form. The only cusps between 0 and $\lambda$ whose reduced form has denominator $\lambda$ are $1 / \lambda$ and $\lambda / \lambda$. Hence any cusp whose reduced form has denominator $\lambda$ is of the form $\left(u \lambda^{2}+1\right) / \lambda$ or $\left(u \lambda^{2}+\lambda\right) / \lambda$ where $u \in \mathbb{Z}$ [LLT1]. The result follows easily from this.

Lemma 8. Let $p$ be a prime in $\mathbb{Z}[\lambda]$ such that $G_{0}(p)$ has $p+1$ inequivalent cusps. Then $N\left(G_{0}(p)\right) / G_{0}(p)$ is a subgroup of $\mathbb{Z}_{2}$.

Proof. Suppose $N\left(G_{0}(p)\right) \neq G_{0}(p)$. For any $A \in N\left(G_{0}(p)\right) \backslash G_{0}(p)$, by Lemmas 1 , $2,3,4$, and $5, A$ is of the form

or (iii) $\quad a b c d \neq 0$.

$$
\text { (i) } A_{1}=\left(\begin{array}{cc}
0 & -1 / \sqrt{p} \\
\sqrt{p} & h \sqrt{p}
\end{array}\right) \text { or } \quad \text { (ii) } A_{2}=\left(\begin{array}{cc}
h \sqrt{p} & 1 / \sqrt{p} \\
-\sqrt{p} & 0
\end{array}\right)
$$

(i) Suppose $A$ takes the form

$$
A=A_{1}=\left(\begin{array}{cc}
0 & -1 / \sqrt{p} \\
\sqrt{p} & h \sqrt{p}
\end{array}\right) .
$$


A simple calculation shows that

$$
A\left(\begin{array}{cc}
1 & 0 \\
p \lambda & 1
\end{array}\right) A^{-1}=\left(\begin{array}{cc}
1-h p \lambda & -\lambda \\
h^{2} p^{2} \lambda & 1+h p \lambda
\end{array}\right) \in G_{0}(p)
$$

This implies that

$$
\frac{-\lambda}{1+h p \lambda}
$$

is a reduced form. In the case $p \neq 2$, by Lemma $7, h=k \lambda$ for some $k$ in $\mathbb{Z}$. Hence

$$
\left(\begin{array}{cc}
1 & 0 \\
k p \lambda & 1
\end{array}\right) A=\left(\begin{array}{cc}
0 & -1 / \sqrt{p} \\
\sqrt{p} & 0
\end{array}\right) \in N\left(G_{0}(p)\right)
$$

It follows that

$$
A G_{0}(p)=\left(\begin{array}{cc}
0 & -1 / \sqrt{p} \\
\sqrt{p} & 0
\end{array}\right) G_{0}(p) .
$$

In the case $p=2$, by Lemma $7, h=k \lambda$ or $1+k \lambda$. It follows easily that

$$
\left(\begin{array}{cc}
1 & 0 \\
2 k \lambda & 1
\end{array}\right) A=\left(\begin{array}{cc}
0 & -1 / \sqrt{2} \\
\sqrt{2} & 0
\end{array}\right) \text { or }\left(\begin{array}{cc}
0 & -1 / \sqrt{2} \\
\sqrt{2} & \sqrt{2}
\end{array}\right) .
$$

We show that the second case is not possible. By [LLT1],

$$
\begin{gathered}
\sigma=\left(\begin{array}{cc}
2 \lambda+1 & -\lambda \\
2 \lambda & -1
\end{array}\right) \in G_{0}(2) . \\
\left(\begin{array}{cc}
0 & -1 / \sqrt{2} \\
\sqrt{2} & \sqrt{2}
\end{array}\right) \sigma\left(\begin{array}{cc}
0 & -1 / \sqrt{2} \\
\sqrt{2} & \sqrt{2}
\end{array}\right)^{-1}=\left(\begin{array}{cc}
* & \lambda \\
* * & 4 \lambda+1
\end{array}\right) \notin G_{0}(2)
\end{gathered}
$$

$(\lambda /(4 \lambda+1)$ is not a reduced form $)$. Hence, the second case is not possible.

It follows that

$$
A G_{0}(2)=\left(\begin{array}{cc}
0 & -1 / \sqrt{2} \\
\sqrt{2} & 0
\end{array}\right) G_{0}(2) .
$$

(ii) Suppose $A$ takes the form

$$
A=A_{2}=\left(\begin{array}{cc}
h \sqrt{p} & 1 / \sqrt{p} \\
-\sqrt{p} & 0
\end{array}\right) .
$$

Since $A_{2}$ is the inverse of $A_{1}$ and $A_{1} G_{0}(p)$ has order 2 in $N\left(G_{0}(p)\right) / G_{0}(p)$,

$$
A_{2} G_{0}(p)=A_{1} G_{0}(p)=\left(\begin{array}{cc}
0 & -1 / \sqrt{p} \\
\sqrt{p} & 0
\end{array}\right) G_{0}(p) .
$$

(iii) Suppose $a b c d \neq 0$. By Lemma $5, b / a$ is an element of $\mathbb{Q}(\lambda)$. Furthermore, if $x / y$ is the reduced form of $-b / a$, then $y$ is a multiple of $p(\tau=p)$. By Leutbecher's Theorem ([L1], [L2]) $-b / a=x / y$ is a cusp of $G$. By Proposition 6(ii) of [LLT1], $G$ contains an element of the form

$$
B=\left(\begin{array}{cc}
x & z \\
y & w
\end{array}\right)
$$

Since $y$ is a multiple of $p, B \in G_{0}(p)$. A direct calculation shows that

$$
\sigma=A B=\left(\begin{array}{cc}
0 & -u^{-1} \\
u & v
\end{array}\right) \in N\left(G_{0}(p)\right)
$$


and $\sigma \infty=0$. By Lemma 4 ,

$$
A B=\left(\begin{array}{cc}
0 & -1 / \sqrt{p} \\
\sqrt{p} & h \sqrt{p}
\end{array}\right)
$$

As above, one has

$$
A G_{0}(p)=A B G_{0}(p)=\left(\begin{array}{cc}
0 & -1 / \sqrt{p} \\
\sqrt{p} & 0
\end{array}\right) G_{0}(p) .
$$

Summing up the above, we have

$$
A G_{0}(p)=\left(\begin{array}{cc}
0 & -1 / \sqrt{p} \\
\sqrt{p} & 0
\end{array}\right) G_{0}(p)
$$

for all $A \in N\left(G_{0}(p)\right) \backslash G_{0}(p)$. This implies that $N\left(G_{0}(p)\right) / G_{0}(p)$ is a subgroup of $\mathbb{Z}_{2}$.

Lemma 9. $r / s \in \mathbb{Q}[\lambda]^{\times}$such that $(r, s)=1$ is equivalent to $\infty$ in $G_{0}(p)$ if and only if $s$ is a multiple of $p$ in $\mathbb{Z}[\lambda]$.

Proof. It is clear that if $r / s$ is equivalent to $\infty$ in $G_{0}(p)$, then $s$ is a multiple of $p$. Conversely, for any $c=x / p y \in \mathbb{Q}[\lambda]$ such that $x \neq 0$ and $(x, p)=1$, let $x^{\prime} / y^{\prime}$ be the reduced form of $c$. By Leutbecher's Theorem ([L1], [L2]) and Proposition 6(ii) of [LLT1], $G$ contains an element of the form

$$
A=\left(\begin{array}{cc}
x^{\prime} & z \\
y^{\prime} & w
\end{array}\right)
$$

Since $(x, p)=1, y^{\prime}$ is a multiple of $p$. This implies that $A \in G_{0}(p)$. Consequently, $c$ is a cusp of $G_{0}(p)$ equivalent to $\infty$.

Theorem 10. Let $p$ be a prime in $\mathbb{Z}[\lambda]$ such that $G_{0}(p)$ has $p+1$ inequivalent cusps. Then $N\left(G_{0}(p)\right)=G_{0}(p)$.

Proof. Suppose not. By Lemma $8, N\left(G_{0}(p)\right) / G_{0}(p) \simeq \mathbb{Z}_{2}$ and

$$
N\left(G_{0}(p)\right)=\left(\begin{array}{cc}
0 & -1 / \sqrt{p} \\
\sqrt{p} & 0
\end{array}\right) G_{0}(p) \cup G_{0}(p) .
$$

This implies that $N\left(G_{0}(G(p))\right.$ has at least 2 cusps.

Let $d$ be a cusp of $G_{0}(p)$ such that $d$ is not equivalent to $\infty$ in $N\left(G_{0}(p)\right) . d \neq 0$ since 0 is equivalent to $\infty$ in $N\left(G_{0}(p)\right)$ by

$$
\left(\begin{array}{cc}
0 & -1 / \sqrt{p} \\
\sqrt{p} & 0
\end{array}\right) .
$$

Write $d=r / s \in \mathbb{Q}[\lambda]^{\times}((r, s)=1)$. Since $d$ is not equivalent to $\infty$ in $G_{0}(p), s$ is not a multiple of $p$ (Lemma 9$)$. However,

$$
\left(\begin{array}{cc}
0 & -1 / \sqrt{p} \\
\sqrt{p} & 0
\end{array}\right) \frac{r}{s}=-\frac{s}{p r}
$$

and $(s, p)=1$; hence $d$ is equivalent to $\infty$ in $N\left(G_{0}(p)\right)$, a contradiction. This completes the proof of Theorem 10 and hence the main theorem. 


\section{REFERENCES}

[AL] A. O. L. Atkin, J. Lehner, Hecke operators on $\Gamma_{o}(m)$, Math. Ann. 185, (1970), $134-160$. MR 42:3022

[C] J. H. Conway, Understanding Groups like $\Gamma_{o}(N)$, Groups, difference sets and the monster (Columbus, Ohio, 1993), Ohio State Univ. Math. Res. Inst. Publ., 4, de Gruyter, Berlin, 1996, 327-343. MR 98b:11041

[CLLT] S. P. Chan, M. L. Lang, C. H. Lim, S. P. Tan, The invariants of the congruence subgroups $G_{0}(P)$ of the Hecke group, Illinois J. of Math. 38 (1994), $636-652$.

[L1] A. Leutbecher, Uber die Heckeschen Gruppen G( $\lambda$ ), Abh. Math. Sem. Hambg. 31 (1967), 199 - 205. MR 37:4018

[L2] A. Leutbecher, Uber die Heckeschen Gruppen $G(\lambda), I I$, Math. Ann. 211 (1974), 63 - 68. MR 50:238

[LT] M. L. Lang, S. P. Tan, Normalizer of the congruence subgroups of the Hecke groups $G_{4}$ and $G_{6}$., (in preparation).

[LLT1] M. L. Lang, C. H. Lim, S. P. Tan, Independent generators for congruence subgroups of Hecke groups, Math. Z. 220 (1995), 569 - 594. MR 96k:11049

[LLT2] M. L. Lang, C. H. Lim, S. P. Tan, Principal congruence subgroups of the Hecke groups, (submitted for publication).

[LN] J. Lehner, M. Newman, Weierstrass Point of $\Gamma_{o}(N)$, Annals of Math. 79 (1964), 360-368. MR 28:5045

[P] L.A. Parson, generalized Kloosterman sums and the Fourier coefficients of cusp forms, Trans. Amer. Math. Soc. 217 (1976), 329 - 350. MR 54:241

[R] D. Rosen, The substitutions of the Hecke group $\Gamma(2 \cos \pi / 5)$, Arch. Math., 46 (1986), 533 - 538. MR 87k:11048

Department of Mathematics, National University of Singapore, Singapore 119260 , RePublic of Singapore

E-mail address: matlml@math.nus.edu.sg 